Original Research

\title{
Neural Response to High and Low Energy Food Images in Anorexia Nervosa
}

Nasim Foroughi ${ }^{1,}{ }^{*}$, Brooke Donnelly ${ }^{2}$, Mark Williams ${ }^{3}$, Sloane Madden ${ }^{4}$, Michael Kohn ${ }^{4}, 5$, Simon Clarke $^{5}$, Perminder Sachdev ${ }^{6}$, Stephen Touyz ${ }^{2,7}$, Phillipa Hay ${ }^{1,8}$

1. School of Medicine, University of Western Sydney, NSW, Australia; E-Mails: n.foroughi@westernsydney.edu.au; P.Hay@westernsydney.edu.au

2. School of Psychology, University of Sydney, NSW, Australia; E-Mails: brookeallana@gmail.com; stephen.touyz@sydney.edu.au

3. Department of Cognitive Science, Macquarie University, Sydney, NSW, Australia; E-Mail: mark.williams@mq.edu.au

4. Department of Psychological Medicine, Sydney Children's Hospital Network Sydney, NSW, Australia; E-Mails: sloane.madden@health.nsw.gov.au; michael.kohn@health.nsw.gov.au

5. Adolescent and Young Adult Medicine, Westmead Hospital, Sydney, NSW, Australia; E-Mail: simon.clarke@sydney.edu.au

6. Discipline of Psychiatry, Faculty of Medicine, University of New South Wales, NSW, Australia; EMail: p.sachdev@unsw.edu.au

7. InsideOut Institute, Charles Perkins Centre, University of Sydney, NSW, Australia

8. Translational Health Research Institute, School of Medicine, Western Sydney University, NSW Australia

* Correspondence: Nasim Foroughi; E-Mail: n.foroughi@westernsydney.edu.au

Academic Editor: Bart Ellenbroek

OBM Neurobiology

2021, volume 5, issue 3

doi:10.21926/obm.neurobiol.2103107
Received: June 01, 2021

Accepted: September 22, 2021

Published: September 30, 2021

\section{Abstract}

To compare neural responses to high and low-energy food images in patients with Anorexia Nervosa (AN) and an age-matched Healthy Control (HC) group. 25 adolescents with AN and $21 \mathrm{HCs}$ completed a diagnostic interview, self-report questionnaires and fMRI, during which they viewed food images evoking responses of disgust, happiness, or fear. Following whole

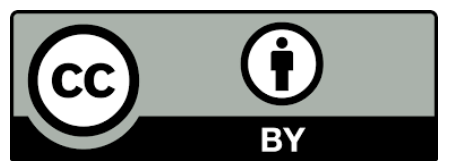

(c) 2021 by the author. This is an open access article distributed under the conditions of the Creative Commons by Attribution License, which permits unrestricted use, distribution, and reproduction in any medium or format, provided the original work is correctly cited. 
brain analyses, neural responses in six regions of interest were examined in a series of between-group contrasts, across the three emotive categories. Compared to the HCs, people in the AN group showed increased responsivity to high-energy (1) disgust images in temporal lobe, frontal lobe, insula, and cerebellum anterior lobe; (2) fear images in occipital lobe, temporal, and frontal lobes and (3) happy images in frontal lobe, cerebellum anterior lobe, sub-lobar, and cuneus. More activity was observed in response to low-energy (1) disgust food images in the temporal lobe, frontal lobe, insula, cerebellum anterior and posterior lobes, parietal lobe, occipital lobe, and limbic lobe; (2) and happy food images in frontal lobes. Few correlations were found with levels of eating disorder symptoms. The findings highlight the emotional impact of diverse high and low-energy foods for people with AN. People without AN may have a better capacity to filter salient from non-salient information relating to the current task when viewing high energy foods. In summary, for those with AN, it would seem their ability to efficiently 'sort-out' information (especially information pertaining to disorderrelevant stimuli such as food images) to complete the task at hand, may be diminished.

\section{Keywords}

Eating disorders; food; emotional response; anorexia nervosa; fMRI

\section{Introduction}

How people with Anorexia Nervosa (AN) emotionally respond to food is a central but incompletely understood psychopathological feature. Research has consistently highlighted that people with a diagnosed Eating Disorder (ED) or sub-threshold symptoms self-report higher negative emotions (e.g. disgust, anxiety, fear) in response to food stimuli compared to Healthy Controls (HCs) [1-5]. Harvey et al. (2002) found that levels of disgust and fear in response to high calorie food images increased with abnormal eating attitudes [6]. In a later study of non-ED women, as eating concerns increased, so too did the degree of fear response to food images [7]. A systematic review [8] and a meta-analysis [9] in people with AN have reported that there is increased activity and an increased hemodynamic response in the emotion-related regions of the brain (frontal, caudate, uncus, insula, and temporal) in response to food and body stimuli. In functional MRI studies there has been a research focus on responses to pleasurable foods [10-14] supporting a paradigm of reduced reward responsivity in people with AN [15]. Thus, findings of increased activity in the cognitive control-related neural circulatory and reduced emotive response to food in appetiterelated brain regions (emotional and reward-related) have been proposed as the core neural mechanisms for the development of AN [16-19]. The study of emotional responsivity in people with AN however, also comprises observed negative emotions such as fear and disgust towards food [20] that may be an illness consequence or maintaining factor in contrast to a predisposing or vulnerability factor.

Whilst an increasing number of studies have examined neural responses to food image stimuli in people with AN, to date studies have had inconsistent findings leaving the neural underpinnings for AN unclear. These inconsistences and a wide range of outcomes were highlighted in a recent further systematic review of functional brain alterations in people with active or recovered AN [10]. The 
observed differences and inconsistencies mainly reflect the methodological variations in the design of the studies including experimental paradigms and sample differences, e.g., the state of satiation of participants. For example, greater activation in response to food stimuli was observed in the emotion-processing regions of the brain of AN patients versus HCs when hungry suggesting a more emotionally arousing response to food stimuli in AN when not satiated [21]. Similarly, increased activation was reported in the caudate in both $\mathrm{AN}$ and AN-recovered patients [22]. However, weaker activation in the parietal cortex was found in people with AN who were satiated relative to HCs [3].

A further source of difference in the research findings is studies at varied stages of AN's onset, recovery, and particular brain processes that may be implicated in adolescence [23]. Eating disorders usually develop during the adolescence years [24] when the brain maturity is incomplete. Emotional and food stimuli processing changes may be more remedial to change. Only two studies have reported findings in adolescents [25, 26]. Killgore et al. [26] reported increased activity in left hippocampus and sub-genual cingulate regions when viewing high-energy foods and increased activity in the fusiform gyrus when viewing low-energy foods. Interestingly, with increased age there was activation of prefrontal cortical regions. Horndasch et al. [25] also found differences between adults and adolescents. In contrast to [26], there was activation of inferior frontal and medial prefrontal gyri and the insula in adolescents when viewing high-energy foods. Additionally, in the cerebellum, when viewing low-energy foods. These results also differed form adults with AN [25]. These adolescent studies also did not report whether the changes correlated with relevant clinical features.

In the light of these inconsistent findings and potentially differential findings in adolescents where the brain is in a state of incomplete maturation, we aimed to further investigate in a sample of adolescents with $A N$, their cerebral response to food stimuli. Further, we included participants who were in a satiated state, thus reducing potential effects from immediate hunger e.g., an exaggerated response to high energy foods. Whilst unknown, it is also possible that people with AN develop conscious mechanisms to facilitate their restricted eating behaviour during hunger. We also included a normal weight control group as the effects of starvation are not known. In addition, in our previous study on this sample we found reduced grey matter volume in brain's frontal, temporal, parietal and occipital cortices and in subcortical areas of the AN group compared to the HCs [27].

To our knowledge, no previous fMRI studies including AN participants have separated pictorial food stimuli into low and high-energy foods and into the three main or primary emotions of happiness, fear and disgust. The separation of food stimuli into these categories, offers a novel investigation and will provide clinically meaningful insight into whether high-energy foods prompt a differential neural response related to low-energy foods, and if this is observed across a range of positive and negative emotions. In the present paper we thus examined the neural response during fMRI to the low-energy and high-energy food stimuli separately, with the results pertaining to each food image category analyzed separately, with a broader consideration of results across low and high-energy conditions, made in the discussion.

We hypothesized that images of high and low-energy food stimuli would evoke a reduced response in these emotion-related brain regions in people with AN compared to the HCs. We further hypothesized that for high-energy foods, the emotional categories would lose their distinctions in the AN group, owing to an overwhelming response evoked, whereas in low-energy foods the AN participants' responses would be closer to the HCs. We also examined the relationship between 
neural activity in response to food images and ED symptoms using beta-value correlations. As this is a novel study, we reported whole-brain exploratory analyses in addition to putative region-ofinterest (ROI) effects such as occipital and parietal-temporal association cortices, in the insula (linked with disgust), amygdala (linked with fear), and medial prefrontal cortex.

\section{Materials and Methods}

\subsection{Recruitment}

Participants with AN were recruited from hospital ED clinics. The HCs consisted of volunteers responding to advertisements placed in educational institutions, advertising in social media networks, and university newsletters. Measurements were collected prior to randomization at baseline and participants $(n=182)$ were recruited as part of a broader study of emotions and food [1]. In exchange for any associated travel costs to attend the assessments participants were reimbursed with a $\$ 30$ gift voucher.

\subsection{Eligibility Criteria}

Underweight in-patient medically stable female participants in their first week of admission (To standardize the disease state) for refeeding $(n=25)$ aged $\geq 14$ years with a DSM-5 AN diagnosis [28, 29] of $<3$ years duration were included. Aged-matched female volunteer without a history of an ED (confirmed by completing diagnostic questionnaires e.g., MINI, EDE-Q) were recruited to the HC group ( $n=21)$. The comprehensive treatment regime has been previously reported [30]. Participants were excluded if they had any contradictions to MRI including claustrophobia, metal implant, heart pacemaker, or pregnancy; major neurological brain impairment, injury, and/or cognitive impairment. Medication or handedness were not recorded.

\subsection{Assessments}

To calculate BMI $\left(\mathrm{kg} / \mathrm{m}^{2}\right)$ percentiles, height and weight were measured using calibrated scales and a stadiometer.

\subsection{Functional Magnetic Resonance Imaging (fMRI) Acquisition}

A GE-3T Signa HDxT (Version 15M4, 8-channel array head coil) fMRI scan of the brain (sagittal orientation) was conducted at Westmead Hospital's imaging center. Functional MRI runs were acquired using a gradient-echo Echo-planar sequence (TR=2s, TE=40ms, $128 \times 128$ matrix, 240mm FOV, 30 slices of $2 \mathrm{~mm}$ thick $+20 \%$ spacing). Prior to the first run, a T1-weighted sequence (spinecho, $256 \times 192$ matrix, 21 slices of $4 \mathrm{~mm}$ thick $+1.5 \mathrm{~mm}$ spacing) was performed to obtain anatomical detail in the same slice planes used for fMRI. A high-resolution T1-weighted sequence was also acquired at the end of each session (Inversion Recovery-prepared, Spoiled Gradient Refocused gradient echo (IRSPGR) $1 \mathrm{~mm} \times 1 \mathrm{~mm}$ in-plane, $1 \mathrm{~mm}$ thick slices) followed by a B0 image. 


\section{5 fMRI Stimuli}

Participants were scanned satiated and hydrated. Three continuous blocks with 96 images of low (e.g., a bunch of English spinach) and high-energy (e.g., a block of Cadbury's chocolate) sweet and savoury food, and neutral non-food objects (e.g., a brick office block, a door, see Image1), were presented at half a second intervals via dual video screen goggles equipped with eye tracking capability. The non-food images $(n=16)$ were embedded within the other types of stimuli and presented randomly. This was done to normalize the data ensuring extraneous variables don't factor into the results. A Rapid Serial Visual Presentation (RSVP) task was used to control for any potential attentional related confounds. Participants were instructed to a tap a buzzer if they saw the same image twice in a row. The food images were further categorized within the low-energy food images, in terms of evoking emotional responses of disgust, happiness or fear. The images were created by author $\mathrm{PH}$ and have been shown to evoke emotions in these categories previously in people with eating disorders [1] and in studies of adolescents [7]. Examples of food images in different low-energy emotive categories include raw baby octopus (disgust), cherries (happy) and fresh chili's (fear). Images were inspected and balanced for complexity by consensus (See Figure 1).

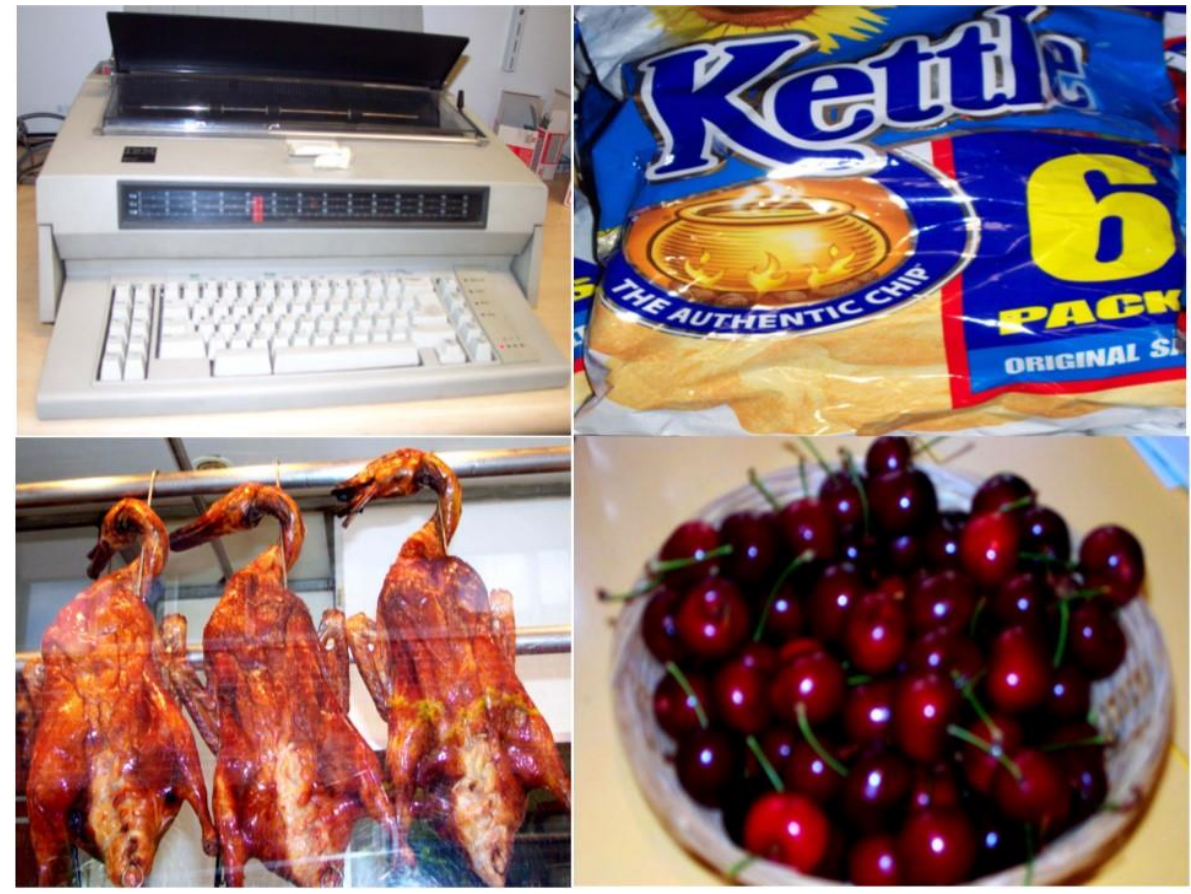

Figure $1 \mathrm{High}$, low, and neutral food/none-food stimuli to evoke responses of disgust, happiness, or fear.

A six-block design was used for presenting the images during the scan ( 2 energy levels (high \& low) $\times 3$ emotions evoked). Each block had 32 images and took 16 minutes to complete. Images were presented in 3 runs of random order. Each run comprised 6 different categories including high and low-energy happiness, disgust, and fear evoking images. Imaging was conducted approximately 30 minutes following a regular meal and took approximately 50 minutes (See Diagram 1). 


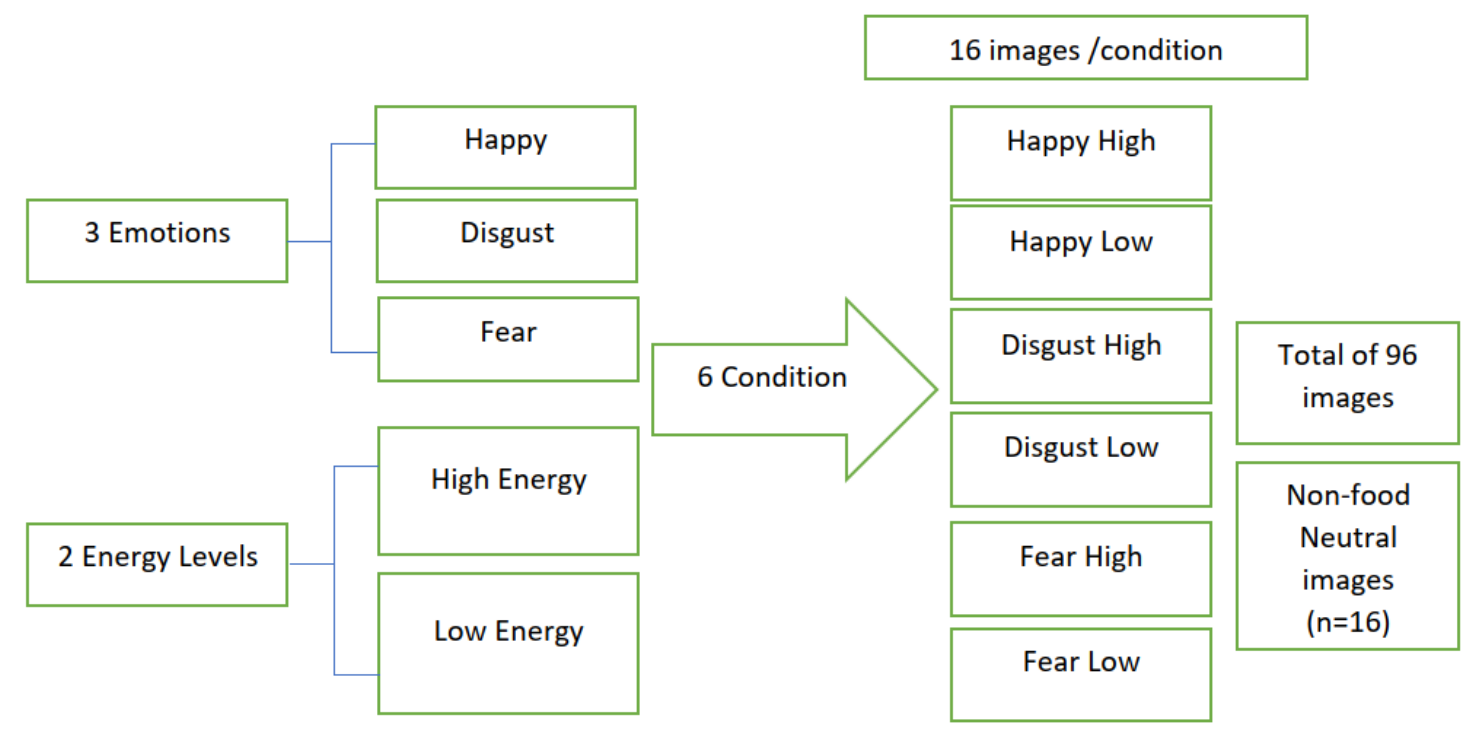

Diagram1 Flow of the 6-block design fMRI stimuli presented to participants.

\section{6 fMRI Data Analysis}

Pre-processing and data analysis were performed via Statistical Parametric Mapping (SPM12) software. The first 4 volumes of each run were removed automatically prior to analysis. Functional data was realigned within scanning runs to correct for head motion using a set of 6 rigid body transformations determined for each image. Each functional run was spatially normalized to the EPI-template supplied with SPM, beginning with a local optimization of the 12-parameters of an affine transformation. These images were then smoothed with a $6 \mathrm{~mm}$ Gaussian filter. The BloodOxygen Level Dependent (BOLD) signal was analysed within each run using a high-pass frequency filter (cut-off 144 seconds). Correlations between scans and epochs were modelled by a standard hemodynamic response function at each voxel. Parameter estimates were obtained for each condition and participant to allow second-level random effects analysis of between-participant variability. Analyses of Variance (ANOVA) was then performed on the statistical maps obtained. The significant clusters (FWE corrected <.05) were independently defined in each hemisphere using WFU_PickAtlas [31].

MATLAB R2017b (MathsWorks) was used in conjunction with SPM12 [32] to perform statistical analyses on $\mathrm{fMRI}$ data. First-level, or individual participant analysis, was completed by generating brain contrast images in each condition of the image stimuli (low-energy: disgust - neutral, fear neutral, happy - neutral) to determine the specific effects of the food image emotive categories. The first-level contrasts were then used in second-level analyses. Second-level, or between-group data analysis, was completed using a full factorial design with two groups (AN and HC) and three values per group (low-energy happy, fear, disgust). Second-level analyses were completed using a combined statistical threshold of $p<.005$ and a voxel cluster size threshold of 10 contiguous voxels, which produces the ideal balance between Type I and Type II error rates [33]. Version 2.5 WFU_Pickatlas was used to complete objective anatomical labelling for any clusters that reached significance [31]. Exploratory whole-brain analyses were completed using the same thresholding guidelines described above [33-35]. 
For B-value correlations non-parametric tests (Pearson correlation) was performed via IBM software (SPSS, version-25). To investigate potential clinical correlates of aberrant activity in different ROI's, beta values for individual subjects were extracted from ROI's using the SPM12 MarsBar toolbox (release 0.44) [36], contrasting (AN- HC) and (HC-AN). To reduce likelihood of Type 1 error ROI's were selected based on the brain regions that had greatest levels of activation for aversive (fear and disgust) emotions and high-energy foods. Clinical characteristics were those with most relevance to food and eating, namely the global EDEQ which reflects eating, shape and weight concern, and dietary restraint. Beta values were correlated with clinical measures to establish any links. Initial univariate analyses were followed by multiple linear regressions to assess whether clinical variables explained for significant variance in the ROI activity. The neural activity in the respective ROI was entered in each model as the dependent variable, while the clinical data were the independent variables. Correction of significance for multiple testing was done to $p<.01$.

\subsection{Assessment Instruments}

Mini International Neuropsychiatry Interview was used to determine current diagnoses modified for DSM-5 AN criteria [28, 29, 37]. The Eating Disorder Examination Questionnaire (EDE-Q) was used to assess eating disorder symptoms over the past 28 days [38]. Subscale scores and a global score are derived and Australian community normative data for these are published [39]. This scale has acceptable internal consistency, test-retest reliability and temporal stability. The 14 -item Hospital Anxiety and Depression Rating Scale (HADS) was used to assess the General psychiatric symptoms of anxiety and depression [40]. It was developed for use in general medical out-patient clinics but is now widely used in clinical practice and research [41] and has been used in non-clinical populations [42]. This scale has adequate test-retest reliability and factor structure in adolescents diagnosed with depressive or anxiety disorders [43].

\subsection{Sample Size}

We aimed for a sample size of 20 per group to detect an effect size of $>.8$, with power $80 \%, p$ $<.05$ one-sided hypothesis [44].

\section{Results}

The majority of the participants were Australian born ( $n=26,56.5 \%)$ and non-smokers of which, $42 \%$ had major depression and $10 \%$ had generalized anxiety disorder (Table 1). Table 2 and 3 present the full results of neural activation contrasted between groups ( $\mathrm{An}-\mathrm{HC} / \mathrm{HC}-\mathrm{AN}$ ) to each of high and low-energy food image conditions, respectively. The main findings of significance are summarized below.

Table 1 Participants' characteristics.

\begin{tabular}{|c|c|c|c|c|c|c|}
\hline Variables & $\begin{array}{l}\text { Anorexia } \\
\text { Nervosa } \\
(n=25)\end{array}$ & $\begin{array}{l}\text { Healthy } \\
\left(n=21^{*}\right)\end{array}$ & Controls & $\mathrm{K}-\mathrm{W} \mathrm{x}^{2}$ & $P<0.05$ & $\begin{array}{l}\text { partial } \\
e t a^{2}\end{array}$ \\
\hline & n (\%) & n (\%) & & $(d f=1)$ & & \\
\hline
\end{tabular}




\begin{tabular}{llllll}
\hline Relationship status* & & & .769 & .380 & - \\
$\quad$ Married & $1(3.8)$ & 0 & - & - & - \\
$\quad$ Single & $25(96.2)$ & $20(100)$ & - & - & - \\
Occupation & & & .009 & .925 & - \\
$\quad$ Employed Full/part time & $2(7.7)$ & $1(5)$ & - & - & - \\
$\quad$ Home maker & $1(3.8)$ & $19(95)$ & - & - & - \\
$\quad$ Student & $21(80.8)$ & 0 & - & - & - \\
Work cover & $2(7.7)$ & 0 & - & - & - \\
& Median (Range) & & & \\
Age (years) & $16(5)$ & $17.5(6)$ & 4.13 & .042 & - \\
& Mean (SD) & & $\mathrm{F}(\mathrm{df}=1)$ & & \\
BMI (kg/m ${ }^{2}$ ) & $16.48(1.8)$ & $23.05(4.03)$ & 51.08 & .001 & .543 \\
EDE-Q & & & & & \\
Global score & $3.64(1.60)$ & $1.46(0.97)$ & 22.39 & .001 & .342 \\
Weight concern & $3.70(1.74)$ & $1.78(1.16)$ & 13.14 & .001 & .234 \\
Shape concern & $4.13(1.76)$ & $2.11(1.4)$ & 13.83 & .001 & .243 \\
Eating Concern & $3.10(1.53)$ & $0.85(1.0)$ & 26.65 & .001 & .383 \\
Restraint & $3.66(1.78)$ & $1.12(1.16)$ & 23.44 & .001 & .353 \\
HADS & & & & & \\
Depression & $9.46(3.97)$ & $5.75(3.94)$ & 8.87 & .005 & .171 \\
Anxiety & $11.88(4.41)$ & $4.35(2.51)$ & 37.04 & .001 & .463 \\
\hline
\end{tabular}

*None of the participants were separated or divorced, SD: Standard Deviation; BMI: Body Mass Index; EDE-Q: Eating Disorder Examination Questionnaire [38]; HADS: Hospital Anxiety and Depression Rating Scale by [40].

Table 2 Neural activation while viewing high energy disgust, fear and happy food images compared to neutral images in Anorexia Nervosa $(n=25)$ and Healthy Controls $(n=21)$.

\begin{tabular}{|c|c|c|c|c|c|c|}
\hline Group contrasts (TD lobe) & Region (TD label) & & & $\begin{array}{l}\text { MINI } \\
\text { coordinates }\end{array}$ & & \\
\hline $\begin{array}{l}\text { High energy disgust }>\text { Neutral } \\
\text { AN }>\text { HC Disgust }\end{array}$ & & $x$ & $\mathrm{Y}$ & Z & $p$ & $\mathrm{~K}_{\mathrm{E}}$ \\
\hline \multirow[t]{2}{*}{ Temporal Lobe } & Sub-Gyral & -36 & -58 & 6 & $<.001$ & 69 \\
\hline & $\begin{array}{l}\text { Superior } \\
\text { Gyrus }\end{array}$ & 40 & -56 & 22 & $<.001$ & 205 \\
\hline \multirow[t]{4}{*}{ Frontal Lobe } & Middle Frontal Gyrus & 32 & 44 & -8 & $<.001$ & 161 \\
\hline & Middle Frontal Gyrus & 30 & 34 & 50 & $<.001$ & 58 \\
\hline & Precentral Gyrus & 44 & 6 & 8 & $<.001$ & 56 \\
\hline & Superior Frontal Gyrus & 32 & 48 & 20 & $<.001$ & 61 \\
\hline \multirow[t]{2}{*}{ Sub-lobar } & Insula & -38 & -26 & 16 & .001 & 44 \\
\hline & Insula & -42 & -40 & 18 & $<.001$ & 73 \\
\hline Cerebellum Anterior Lobe & Cerebellar Lingual & 2 & -46 & -24 & .002 & 39 \\
\hline \multicolumn{7}{|c|}{ High energy disgust $>$ Neutral HC $>$ AN Disgust } \\
\hline Occipital Lobe & Middle Occipital Gyrus & 36 & -92 & 2 & $<.001$ & 388 \\
\hline
\end{tabular}




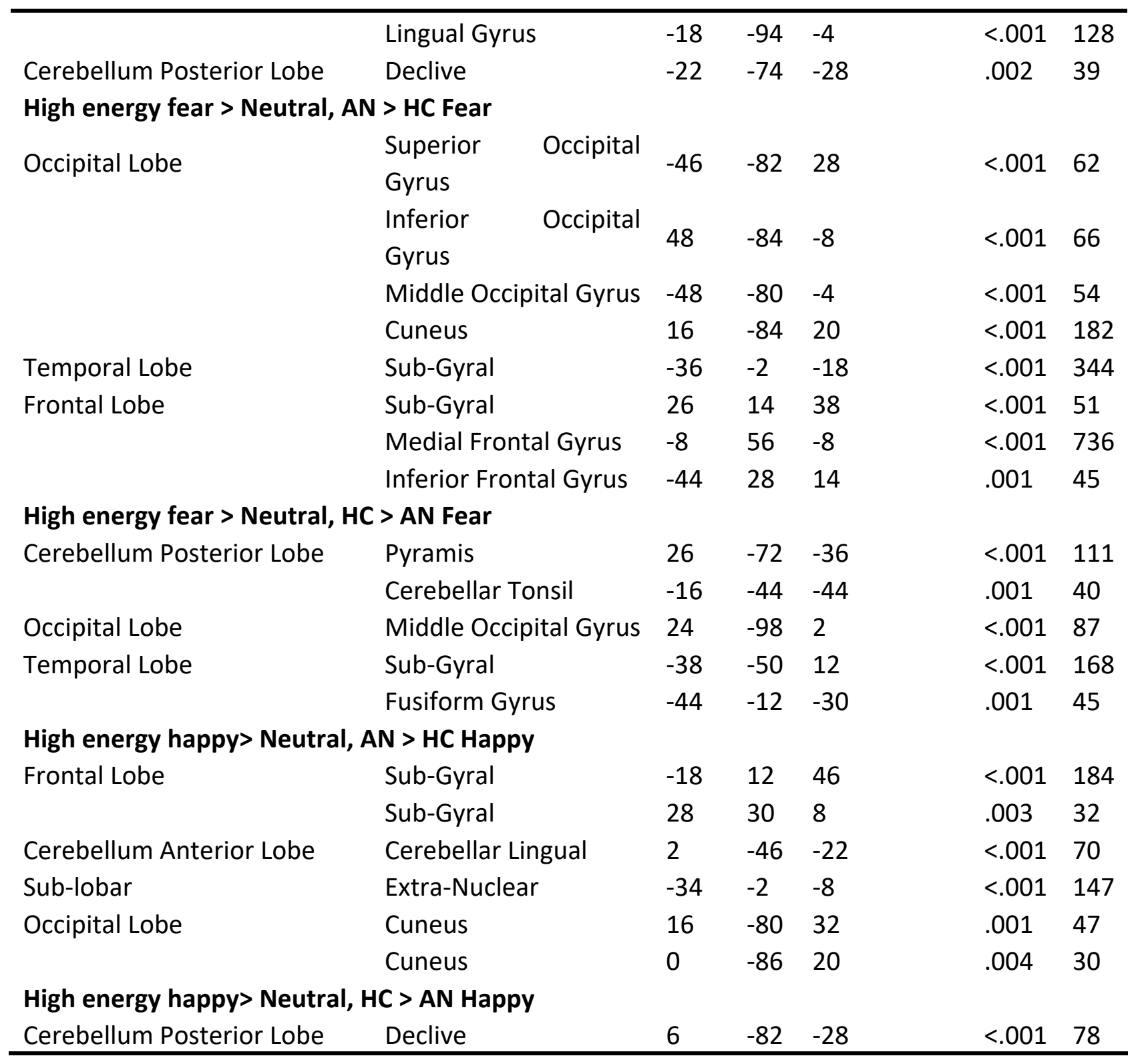

Anorexia Nervosa: AN; Healthy Controls: HCs; TD: Talaraich Daemon neurological database; MNI: Montreal Neurological Institute coordinates; kE: Voxel size.

Table 3 Neural activation while viewing low energy disgust and happy food images compared to neutral images in Anorexia Nervosa $(n=25)$ and Healthy Controls $(n=21)$.

\begin{tabular}{|c|c|c|c|c|c|c|c|}
\hline \multirow{2}{*}{$\begin{array}{l}\text { Group Contrasts (TD lobe) } \\
\text { Low energy disgust > Neutral } \\
\text { AN > HC Disgust }\end{array}$} & \multicolumn{5}{|c|}{ Region (TD label) } & \multicolumn{2}{|c|}{$\begin{array}{l}\text { MINI } \\
\text { coordinate }\end{array}$} \\
\hline & & & $x$ & $Y$ & Z & $p$ & $\mathrm{~K}_{\mathrm{E}}$ \\
\hline \multirow[t]{2}{*}{ Temporal Lobe } & $\begin{array}{l}\text { Superior } \\
\text { Gyrus }\end{array}$ & Temporal & -40 & -48 & 18 & $<.001$ & 158 \\
\hline & $\begin{array}{l}\text { Middle } \\
\text { Gyrus }\end{array}$ & Temporal & 50 & -2 & -20 & $<.001$ & 81 \\
\hline Frontal Lobe & $\begin{array}{l}\text { Superior } \\
\text { Gyrus }\end{array}$ & Frontal & 44 & 38 & 38 & $<.001$ & 110 \\
\hline
\end{tabular}




\begin{tabular}{|c|c|c|c|c|c|c|}
\hline & Sub-Gyral & 26 & -12 & 40 & $<.001$ & 51 \\
\hline & Sub-Gyral & 28 & 34 & 4 & $<.001$ & 71 \\
\hline & Sub-Gyral & 24 & -30 & 42 & $<.001$ & 59 \\
\hline & Inferior Frontal Gyrus & -46 & 20 & -6 & .003 & 29 \\
\hline & $\begin{array}{l}\text { Inferior Parietal } \\
\text { Lobule }\end{array}$ & 36 & -30 & 28 & $<.001$ & 81 \\
\hline & Precentral Gyrus & -26 & -32 & 62 & $<.001$ & 128 \\
\hline \multirow[t]{6}{*}{ Sub-lobar } & Insula & 38 & 0 & 14 & $<.001$ & 304 \\
\hline & Insula & -40 & -4 & 12 & $<.001$ & 129 \\
\hline & Insula & 36 & -42 & 18 & $<.001$ & 115 \\
\hline & Insula & -38 & -26 & 18 & $<.001$ & 139 \\
\hline & Insula & 48 & -34 & 20 & .002 & 31 \\
\hline & Extra-Nuclear & -34 & -4 & -8 & .002 & 33 \\
\hline Cerebellum Anterior Lobe & Cerebellar Lingual & 2 & -42 & -18 & $<.001$ & 59 \\
\hline Cerebellum Posterior Lobe & Cerebellar Tonsil & -20 & -62 & -42 & $<.001$ & 123 \\
\hline Parietal Lobe & Supramarginal Gyrus & 44 & -52 & 34 & .005 & 25 \\
\hline Occipital Lobe & Sub-Gyral & 38 & -62 & -4 & $<.001$ & 45 \\
\hline Limbic Lobe & Posterior Cingulate & 24 & -70 & 8 & .002 & 34 \\
\hline \multicolumn{7}{|c|}{ Low energy happy > Neutral, AN > HC Happy } \\
\hline \multirow[t]{4}{*}{ Frontal Lobe } & Inferior Frontal Gyrus & 44 & 42 & 10 & $<.001$ & 227 \\
\hline & Middle Frontal Gyrus & 32 & 16 & 52 & $<.001$ & 79 \\
\hline & Middle Frontal Gyrus & 28 & -8 & 60 & $<.001$ & 56 \\
\hline & Sub-Gyral & 24 & 24 & 30 & .001 & 47 \\
\hline \multicolumn{7}{|c|}{ Low energy happy > Neutral, HC > AN Happy } \\
\hline \multirow[t]{2}{*}{ Cerebellum Posterior Lobe } & Declive & 8 & -84 & -28 & $<.001$ & 111 \\
\hline & $\begin{array}{l}\text { Inferior Semi-Lunar } \\
\text { Lobule }\end{array}$ & 16 & -62 & -50 & .001 & 46 \\
\hline Occipital Lobe & Cuneus & 20 & -98 & 0 & .001 & 45 \\
\hline
\end{tabular}

Anorexia Nervosa: AN; Healthy Controls: HC; TD: Talairach Daemon; MNI: Montreal Neurological Institute coordinates; kE: Voxel size.

\subsection{High-Energy}

\subsubsection{Disgust $>$ Neutral}

AN> HC: high-energy disgust images led to significantly greater activation within the temporal Lobe (sub-gyral and superior temporal gyrus), frontal lobe (middle and superior frontal gyrus and precentral gyrus), insula, and sub lobar (cerebellar lingual). HC>AN: greater activation in HCs, within occipital lobe (middle occipital gyrus, lingual gyrus) and cerebellum posterior lobe (declive). 

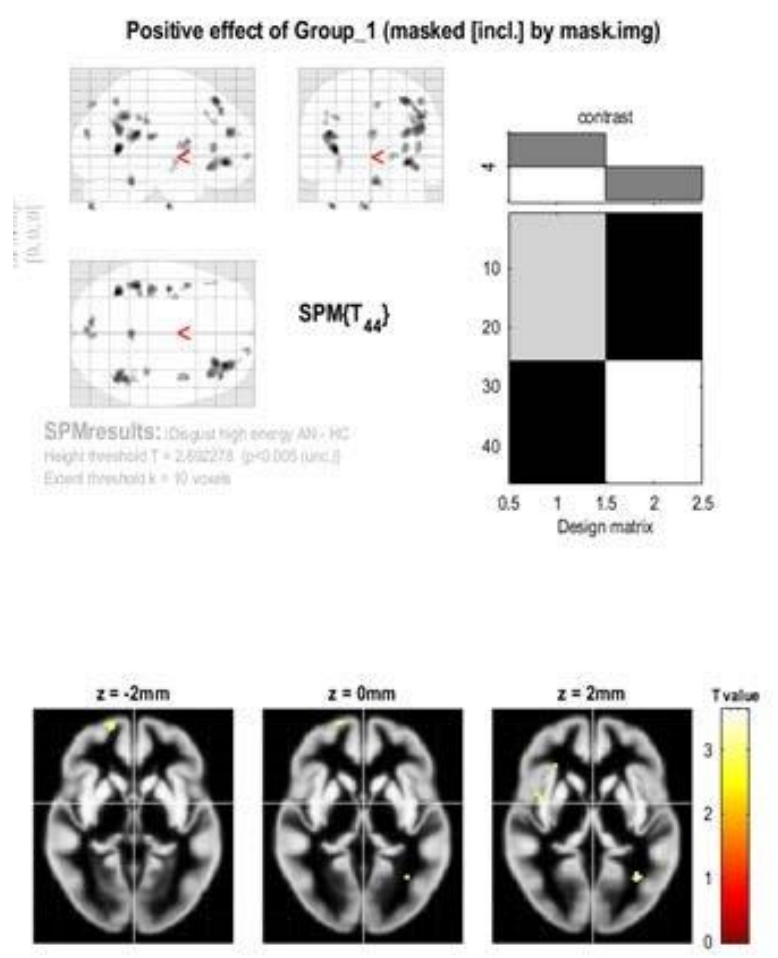

Figure 2 Positive effect of group (high energy disgust AN-HC).

\subsubsection{Fear $>$ Neutral}

AN> HC: greater activation within occipital lobe (superior, inferior and middle occipital gyrus and cuneus), temporal lobe (sub-gyral), and frontal lobe (sub-gyral, medial and inferior frontal gyrus). $\mathrm{HC}>\mathrm{AN}$ : greater activation in $\mathrm{HCs}$, in cerebellum posterior lobe (pyramis, cerebellar tonsil), occipital lobe (middle occipital gyrus), and temporal lobe (sub-gyral, fusiform gyrus).
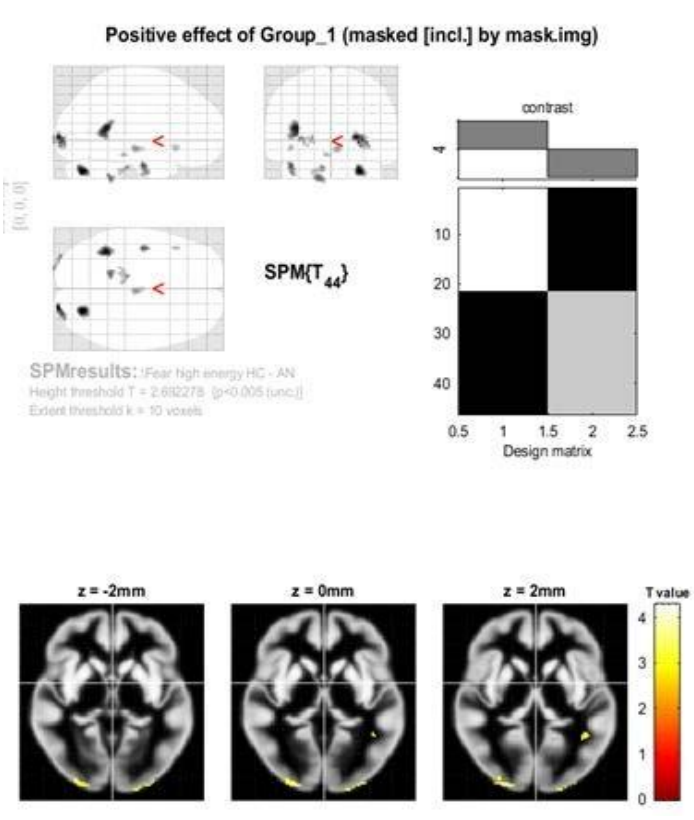

Figure 3 Positive effect of group (high energy fear AN-HC). 


\subsubsection{Happy > Neutral}

AN> HC: greater activation in frontal lobe (sub-gyral), cerebellum anterior lobe (cerebellar lingual), sub-lobar (extra-nuclear), and occipital lobe (cuneus). HC>AN: greater activation in cerebellum posterior lobe (declive).
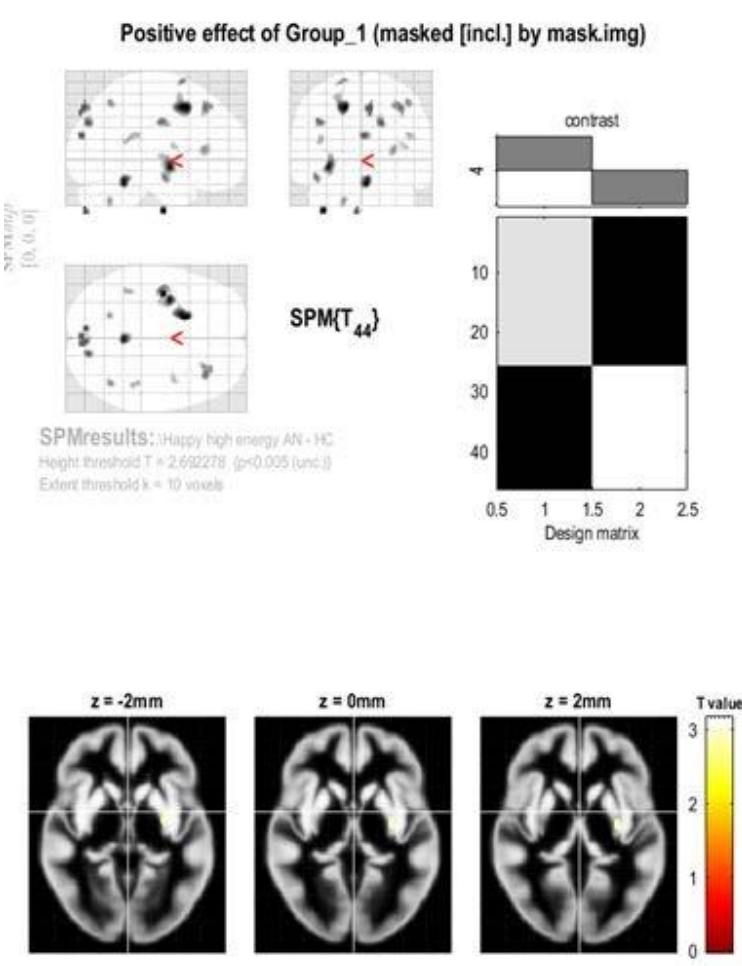

Figure 4 Positive effect of group (High energy happy AN-HC).

\subsection{Low-Energy}

\subsubsection{Disgust $>$ Neutral}

AN> HC: greater activation in temporal lobe (superior and middle temporal gyrus), frontal lobe (superior frontal, sub gyral, inferior frontal gyral, inferior parietal lobule, precentral gyrus), insula, sub-lobar (extra-nuclear), cerebellum anterior lobe (cerebellar lingual), cerebellum anterior lobe (cerebellar tonsil), parietal lobe (supramarginal gyus), occipital lobe (sub-gyral), limbic lobe (posterior cingulate). HC>AN: no ROI or whole-brain exploratory differences evoked in this condition. 

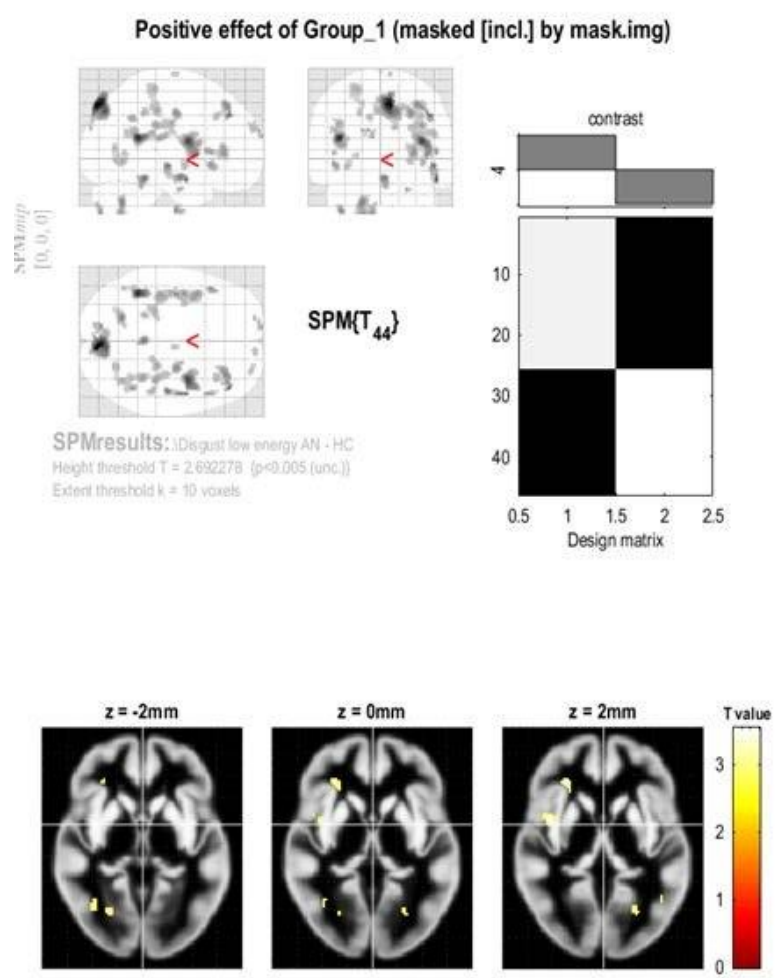

Figure 5 Positive effect of group (low energy disgust AN-HC).

\subsubsection{Fear $>$ Neutral}

AN > HC: no ROI or whole-brain exploratory differences evoked in this condition nor for HC>AN.
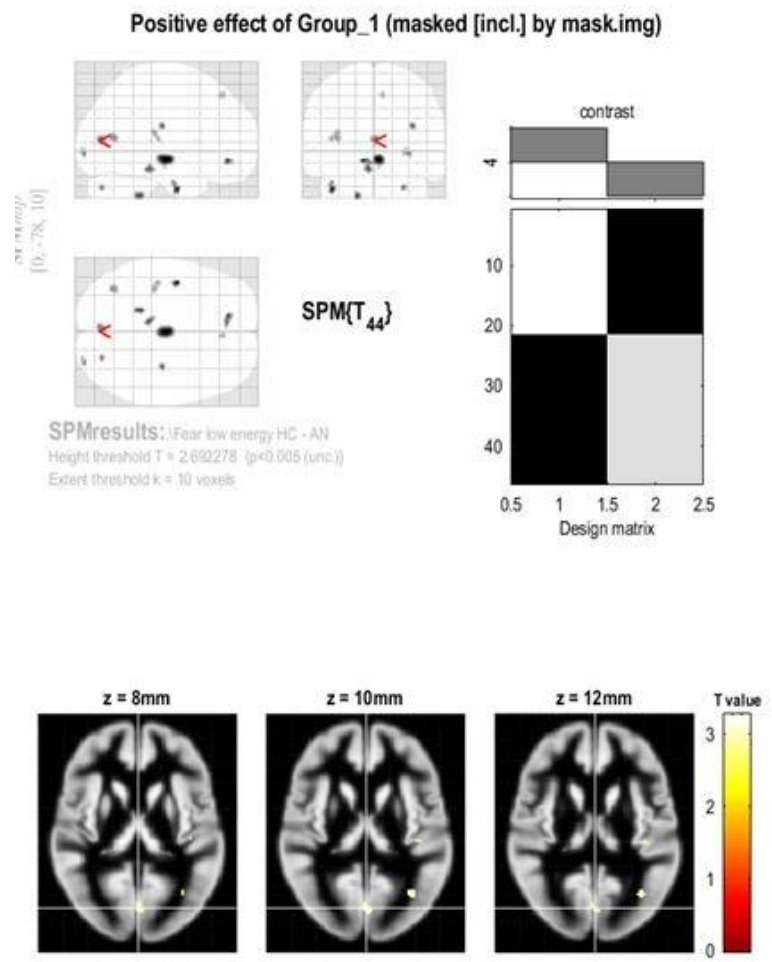

Figure 6 Positive effect of group (low energy Fear AN-HC). 


\subsubsection{Happy > Neutral}

AN> HC: greater activation in frontal lobe (inferior and middle frontal gyrus and sub-gyral). $\mathrm{HC}>\mathrm{AN}$ : greater activation in cerebellum posterior lobe (declive, inferior semi-lunar lobule), occipital lobe (cuneus).

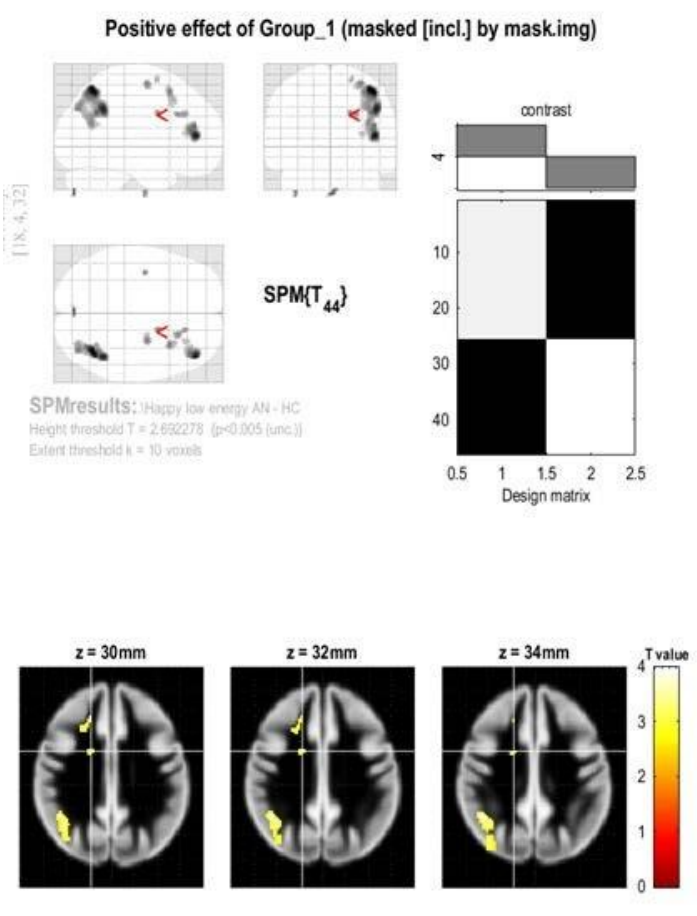

Figure 7 Positive effect of group (low energy happy AN-HC).

\subsection{Correlations}

A range of ROI's were associated with changes in the brain's neural activity in participants with AN across low and high-energy image conditions. On inspection of these results, a subset of ROI's was identified with greater responses than other regions in this cohort. Regarding high-energy images, the temporal lobe, frontal lobe, insula, and cerebellum anterior lobe were hyperactive; however, occipital and cerebellum posterior lobe were hypoactive to viewing disgust images in AN. Viewing fear images, occipital lobe, temporal and frontal lobe were hyperactive while the cerebellum posterior lobe, occipital and temporal lobes were hypoactive. The frontal lobe, cerebellum anterior lobe, sub-lobar, and occipital lobe were hyperactive, while cerebellum posterior lobe was hypoactive in happy category in AN. Viewing low-energy disgust images, the temporal lobe, frontal lobe, insula, cerebellum anterior and posterior lobes, parietal lobe, occipital lobe, and limbic lobe were hyperactive. Finally, viewing happy images, the frontal lobe was hyperactive, while cerebellum posterior lobe and occipital lobe were hypoactive in AN. Therefore, we calculated correlations between EDE-global score as a clinical variable and neural activity in all three ROI's and in all conditions including the brain regions mentioned above. B-values of temporal, frontal, and occipital regions' ROI's were selected as the dependent variables in the regression with EDE-global score as the independent variable. Few correlations were found (Table 4). 
Table 4 Correlations between eating disorder symptoms (Global EDEQ) and areas of activation in response to viewing high and low-energy food.

\begin{tabular}{llll}
\hline & Region (TD label) & $r_{s}$ & $p$ \\
\hline High energy disgust & & & \\
HC>AN & Middle Occipital Gyrus / Occipital Lobe & .305 & .039 \\
& Lingual Gyrus / Occipital Lobe & .249 & .095 \\
& Insula sublobar voxel 44 & .277 & .063 \\
& Insular / Sub-Lobar 73 & .385 & .008 \\
& Precentral Gyrus_FrontalLobe & .287 & .053 \\
High energy fear & Sub-Gyral / Temporal Lobe & .336 & .022 \\
HC $>$ AN & & & \\
& Sub-Gyral / Temporal Lobe & .371 & .011 \\
Low energy disgust & .291 & .050 \\
AN>HC & Middle Occipital Gyrus / Occipital Lobe & -.272 & .068 \\
& Fusiform Gyrus / Temporal Lobe & & \\
& & .317 & .032 \\
& Cerebellar Lingual / Cerebellum Anterior Lobe & .378 & .061 \\
& Insula / Sub-Lobar voxels 115 & .432 & .003 \\
& Middle Temporal Gyrus / Temporal Lobe & .338 & .022 \\
\hline
\end{tabular}

$A N=$ anorexia nervosa; $H C=$ healthy control; TD: Talairach Daemon; Significant findings are in bold.

The Children's Hospital at Westmead and Western Sydney University Human Research Ethics Committees approved the study (Reference No. HREC/13/SCHN/385 and H6644, respectively). All participants and their parents/guardians as appropriate provided informed consent. Professor Phillipa Hay is the principal investigator of this study.

\section{Discussion}

We investigated the neural responses to high and low-energy food images across a range of emotion provoking foods in people with AN compared to a $\mathrm{HC}$ group. The association of neural activity with overall composite score of eating disorder symptoms (dietary restraint, and eating, weight and shape preoccupations) was also examined. AN participants showed greater activation in response to high-energy (1) disgust images in temporal, frontal, sub-lobar (insula), and cerebellum anterior lobe, (2) fear images in occipital, temporal, and frontal lobes, and (3) happy images in frontal, cerebellum, sub-lobar, anterior lobe, and cuneus. Similar pattern of activation was observed in response to low-energy (1) disgust images in temporal, frontal, sub-lobar (insula), cerebellum anterior and posterior lobes, parietal and occipital lobes, and limbic lobe and (2) happy images in frontal lobe. No significant differences were observed in response to low-energy fear images.

In AN participants compared to HCs, we found a wide range of areas of increased activation in response to images associated with aversive emotions, namely disgust, and only fear in high energy foods. Our findings are partly consistent with previous reviews and meta-analysis by [8, 9] where viewing high-calorie disgust images evoked an increased neural activation in insula. The findings of 
increased activation in insula in the disgust condition to both high and low energy foods are consistent with previous research that has implicated the insula as a region that integrates the interoceptive states into decision-making process. Thus, people with an AN may be particularly sensitised to feelings of disgust underpinning a learned negative response when confronted with foods known to evoke this emotion $[45,46]$. This supports a possible association between fear and disgust of high energy foods and activation patterns in AN.

Findings of the current study only in part support the results of a systematic review by [8] in which HCs showed greater activation in occipital cortex and inferior parietal lobule compared to AN participants $[3,5]$. However, some studies have not shown greater activation in HCs compared to ANs in specific areas of the brain [3-5] and in this study participants with AN had higher activation of the visual cortex (occipital lobe) to high energy happy and fear foods compared to the HCs. The observed differences may reflect the methodological variations in the design of the studies, including studies of not satiated participants [3], different experimental paradigms such as instructing participants to think about their perception of hunger when viewing the images [47] and sample differences (e.g. adults vs. adolescents). However, satiation status alone is insufficient to explain differences as enhanced activation in visual areas of the brain (e.g. occipital cortex) in response to motivationally relevant images (food and tools) have been reported by other investigators in HCs when not satiated [48-50] or as in the Uher et al. studies [4, 5] where participants were in an intermediate state between hunger and satiety. Further research is needed to determine the extent of reduced food-related somatosensory processing in people with AN when satiated and when not satiated.

The present study does not support the hypothesis that people with AN had less activation of frontal-limbic pleasure pathways towards high and low-energy happy foods [51]. There was also less activity of the visual cortes towards high energy (but not low energy) happy foods. It contrasts with our previous research that found quantitative and qualitative lower levels of subjectively reported happiness in people with EDs, including AN when viewing such food images [1, 7]. It may be that there is reduced pleasure, but this was not detected due to the effects from increased aversive emotions towards all foods, and in particular, high-energy foods.

The findings of current study may be modulated in part by unknown effects of starvation in these participants with AN, such as widespread reduced grey matter volume compared to the HCs [27]. Reduced regional brain volume may indicate reduced activity in response to food stimuli, or increased activity due to reduced inhibitory mechanisms. However, these findings only partially matched the results of the current fMRI study. For example, areas such as parietal lobe (sub-gyral) did not show greater activation in the HCs, but showed reduced volume in another study by our group [27].

It is unclear how much the findings in the present study may relate to clinical severity or specific clinical features as there were few significant correlations between ED symptoms and ROI's. Further, the correlations were not high except for one region (High energy disgust Insular / Sub-Lobar <.01), and this may have been due to a Type II error given the large number of tests conducted.

Limitations of this study were that men were excluded, medications, illness duration, and handedness were not recorded for all participants in this sample. The sample size in each group $(\mathrm{AN}=25, \mathrm{HCS}=21$ ) exceeded power requirements and we found highly significant between group differences. However, increasing the sample may have improved our ability to detect wider results in relation to beta value correlations with other clinical variables. The illness severity and duration 
in AN [52] and in bipolar depression has negative effects on cognitive performance [53]. Although, the illness duration of more than 3 years was an exclusion criterion for the current study, we did not control for duration in our statistical model. Further, approximately $42 \%$ of the participants were suffering from major depression, however mood status was not found to explain the emotional responses of people with eating disorders to similar food images in a previous study from our group [54]. Longitudinal research in people with AN post-weight recovery is needed to further examine putative effects of starvation.

Whilst definitive conclusions cannot be drawn, for those with AN, the findings suggest their ability to efficiently 'sort-out' information (especially information pertaining to disorder-relevant stimuli such as food images) to complete the task at hand, may be diminished. It provides some neuro-biological understanding of the high levels of distress people with AN experience, particularly in the early phases of refeeding. It supports an understanding for the slow return and graded exposure to a wide range of food consumption as used in cognitive behavioral treatments for EDs (e.g., Fairburn 2008) [55] and the judicious use of antipsychotic medication to reduce arousal during early refeeding and their possible benefits in the treatment of AN [56].

\section{Conclusions}

The present study supported previous research in finding various patterns of neural activation in response to high and low-energy food images, particularly in young women with AN compared to $\mathrm{HCs}$, when satiated. Further research in larger samples also examining responses when hungry, and with a longitudinal follow-up before and after weight regain is needed. This may offer broader understandings and potential clinical applications. Our findings do highlight the importance of the arousal of primary emotive responses particularly disgust evoking foods in people with EDs.

\section{Acknowledgments}

The Authors would like to acknowledge the valuable time and effort of the volunteers who have participated in this study.

\section{Author Contributions}

PH \& NF conceived the idea, developed the theory, and performed the computations. MW verified the analytical methods. All authors discussed the results and contributed to the final manuscript.

\section{Funding}

This project had no external funding.

\section{Competing Interests}

$\mathrm{PH}$ receives sessional fees and lecture fees from the Australian Medical Council, Therapeutic Guidelines publication, and New South Wales Institute of Psychiatry and royalties from Hogrefe and Huber, McGraw Hill Education, and Blackwell Scientific Publications. She has received research grants from the NHMRC and ARC. She is the Deputy Chair of the National Eating Disorders 
Collaboration Steering Committee in Australia (2012) and Member of the ICD-11 Working Group for Eating Disorders (2012) and was Chair Clinical Practice Guidelines Project Working Group (Eating Disorders) of RANZCP (2012-2015). She has prepared a report under contract for Shire Pharmaceuticals (July 2017). All views in this paper are her own. NF has no conflict of interest. She has received research grants from the NHMRC. MW has no conflict of interest. ST is in receipt of royalties for book/chapter publications from Hogrefe and Huber, Mc Graw Hill and Routledge. He chairs the Shire Australian Binge Eating Disorder Advisory Committee and has received honoraria for commissioned reports, travel grants and a research grant from Shire. He is a mental health advisor to the Commonwealth Department of Veteran's Affairs. SM, was a member of the Shire Australian Binge Eating Disorder Advisory Committee 2014-15. He has received research grants from the NHMRC and ARC. He is a member of the National Eating Disorders Collaboration Steering Committee in Australia (2010 - present) and was a member of the Clinical Practice Guidelines Project Working Group (Eating Disorders) of RANZCP (2012-2015). MK is a member of the Shire Australian Binge Eating Disorder Advisory Committee and has received honoraria for commissioned reports, travel grants and a research grant from the Shire. SK has received travel grants and fees from Shire for lectures and conferences associated with ADHD. PS has no conflict of interest.

\section{References}

1. Foroughi N, Madden S, Clarke S, Kohn M, Donnelly B, Touyz S, et al. Do emotional responses to food images differ within different types of eating disorders? Australas Psychiatry. 2020; 28: 128-133.

2. Rodríguez S, Mata JL, Lameiras M, Fernández MC, Vila J. Dyscontrol evoked by erotic and food images in women with bulimia nervosa. Eur Eat Disord Rev. 2007; 15: 231-239

3. Santel S, Baving L, Krauel K, Munte F, Rotte M. Hunger and satiety in anorexia nervosa: fMRI during cognitive processing of food pictures. Brian Res. 2006; 1114: 138-148.

4. Uher R, Brammer MJ, Murphy T, Campbell IC, Ng VW, Williams SC, et al. Recovery and chronicity in anorexia nervosa: Brain activity associated with differential outcomes. Biol Psychiatry. 2003; 54: 934-942.

5. Uher R, Murphy T, Brammer M, Dalgleish T, Phillips ML, Ng VW, et al. Medial prefrontal cortex activity associated with symptom provocation in eating disorders. Am J Psychiatry. 2004; 161: 1238-1246.

6. Harvey T, Troop NA, Treasure JL, Murphy F. Fear, disgust, and abnormal eating attitudes: A preliminary study. Int J Eat Disord. 2002; 32: 213-218.

7. McNamara C, Hay $P$, Katsikitis $M$, Chur-Hansen A. Emotional responses to food, body dissatisfaction and other eating disorder features in children, adolescents and young adults. Appetite. 2008; 50: 102-109.

8. Giel KE, Teufel M, Friederich HC, Hautzinger M, Enck P, Zipfel S. Processing of pictorial food stimuli in patients with eating disorders-A systematic review. Int J Eat Disord. 2011; 44: 105117.

9. Zhu Y, Hu X, Wang J, Chen J, Guo Q, Li C, et al. Processing of food, body and emotional stimuli in Anorexia Nervosa: A systematic review and meta-analysis of functional magnetic resonance imaging studies. Eur Eat Disord Rev. 2012; 20: 439-450. 
10. Fuglset TS, Inge Landr $\varnothing \mathrm{NI}$, Reas DL, R $\varnothing$. Functional brain anorexia nervosa: A scoping review. J Eat Disord. 2016; 4: 1-13.

11. Cowdrey FA, Park RJ, Harmer CJ, McCabe C. Increased neural processing of rewarding and aversive food stimuli in recovered Anorexia Nervosa. Biol Psychiatry. 2011; 70: 736-743.

12. Ellison Z, Foong J, Howard R, Bullmore E, Williams E, Treasure J. Functional anatomy of calorie fear in anorexia nervosa. Lancet. 1998; 352: 1192.

13. Holsen LM, Lawson E, Blum J, Ko E, Makris N, Fazeli PK, et al. Food motivation circuitry hypoactivation related to hedonic and nonhedonic aspects of hunger and satiety in women with active anorexia nervosa and weight-restored women with anorexia nervosa. J Psychiatry Neurosci. 2012; 37: 322-332.

14. Oberndorfer TA, Frank GK, Simmons AN, Wagner A, McCurdy D, Fudge JL, et al. Altered insula response to sweet taste processing after recovery from Anorexia and Bulimia Nervosa. Am J Psychiatry. 2013; 170: 1143-1151.

15. Steinglass JE, Berner LA, Attia E. Cognitive neuroscience of eating disorders. Psychiatr Clin. 2019; 42: 75-91.

16. Friederich HC, Wu M, Simon JJ, Herzog W. Neurocircuit function in eating disorders. Int J Eat Disord. 2013; 46: 425-432.

17. Kaye W, Wierenga CE, Bailer UF, Simmons AN, Bischoff-Grethe A. Nothing tastes as good as skinny feels: The neurobiology of anorexia nervosa. Trends Neurosci. 2013; 36: 110-120.

18. Kaye WH, Fudge JL, Paulus $M$. New insights into symptoms and neurocircuit function of anorexia nervosa. Nat Rev Neurosci. 2009; 10: 573-584.

19. Klein DA, Schebendach JE, Gershkovich M, Smith GP, Walsh BT. Modified sham feeding of sweet solutions in women with anorexia nervosa. Physiol Behav. 2010; 101: 132-140.

20. Hildebrandt T, Grotzinger A, Reddan M, Greif R, Levy I, Goodman W, et al. Testing the disgust conditioning theory of food-avoidance in adolescents with recent onset anorexia nervosa. Behav Res Ther. 2015; 71: 131-138.

21. Mehler PS, Brown C. Anorexia nervosa-Medical complications. J Eat Disord. 2015; 3: 1-8.

22. Sanders N, Smeets PA, van Elburg AA, Danner UN, van Meer F, Hoek HW, et al. Altered foodcue processing in chronically ill and recovered women with anorexia nervosa. Front Behav Neurosci. 2015; 9. doi: 10.3389/fnbeh.2015.00046.

23. Joos AA, Saum B, van Elst LT, Perlov E, Glauche V, Hartmann A, et al. Amygdala hyperreactivity in restrictive anorexia nervosa. Psychiatry Res. 2011; 191: 189-195

24. Fairburn CG, Harrison P. Eating disorders. Lancet. 2003; 361: 407-416.

25. Horndasch S, Roesch J, Forster C, Dörfler A, Lindsiepe S, Heinrich H, et al. Neural processing of food and emotional stimuli in adolescent and adult anorexia nervosa patients. PLoS One. 2018; 13: e0191059.

26. Killgore WD, Yurgelun-Todd DA. Developmental changes in the functional brain responses of adolescents to images of high and low-calorie foods. Dev Psychobiol. 2005; 47: 377-397.

27. Martin Monzon B, Henderson LA, Madden S, Macefield VG, Touyz S, Kohn MR, et al. Grey matter volume in adolescents with anorexia nervosa and associated eating disorder symptoms. Eur J Neurosci. 2017; 46: 2297-2307.

28. American Psychiatric Association. Diagnostic and statistical manual of mental disorders, Fifth Edition. Washington, DC: American Psychiatric Association; 2013. 
29. Sheehan D, Janavs J, Baker R, Harnett-Sheehan K, Knapp E, Sheehan M. Mini international neuropsychiatric interview, English Version 5.0.0 USA. 2005.

30. Madden S, Miskovic-Wheatley J, Wallis A, Kohn M, Lock J, Le Grange D, et al. A randomized controlled trial of in-patient treatment for anorexia nervosa in medically unstable adolescents. Psychol Med. 2015; 45: 415-427

31. Maldjian JA, Laurienti PJ, Kraft RA, Burdette JH. An automated method for neuroanatomic and cytoarchitectonic atlas-based interrogation of fMRI data sets. Neurolmage. 2003; 19: 12331239.

32. Neuroimaging W. Statistical Parametric Mapping (SPM12). Institute of Neurology, University College London. 2013.

33. Lieberman MD, William AC. Type I and Type II error concerns in fMRI research: Re-balancing the scale. Soc Cogn Affect Neurosci. 2009; 4: 423-428.

34. Poldrack RA. Region of interest analysis for fMRI. Soc Cogn Affect Neurosci. 2007; 2: 67-70.

35. Saxe R, Brett $M$, Kanwisher $N$. Divide and conquer: A defense of functional localizers. Neurolmage. 2006; 30: 1088-1096.

36. Brett $M$, Anton JL, Valabregue R, Poline JB. Region of interest analysis using the MarsBar toolbox for SPM 99. Neurolmage. 2002; 16: S497.

37. Sheehan DV, Lecrubier Y, Sheehan KH, Janavs J, Weiller E, Keskiner A, et al. The validity of the Mini International Neuropsychiatric Interview (MINI) according to the SCID-P and its reliability. Eur Psychiatry. 1997; 12: 232-241.

38. Beglin SJ, Fairburn CG. Evaluation of a new instrument for the detection of eating disorders in community samples. Psychiatry Res. 1992; 44: 191-201.

39. Mond JM, Hay PJ, Rodgers B, Owen C. Eating Disorder Examination Questionnaire (EDE-Q): Norms for young adult women. Behav Res Ther. 2006; 44: 53-62.

40. Zigmond AS, Snaith RP. The hospital and anxiety and depression scale. Acta Psychiatr Scand. 1983; 67: 361-370.

41. Herrmann C. International experiences with the Hospital Anxiety and Depression Scale - A review of validation data and clinical results. J Psychosom Res. 1997; 42: 17-41.

42. Crawford JR, Henry JD, Crombie C, Taylor EP. Normative data for the HADS from a large nonclinical sample. Br J Clin Psychol. 2001; 40: 429-434.

43. White D, Leach $C$, Sims R, Atkinson M, Cottrell D. Validation of the hospital anxiety and depression scale for use with adolescents. Br J Psychiatry. 1999; 175: 452-454.

44. Desmond JE, Glover GH. Estimating sample size in functional MRI ( $\mathrm{fMRI}$ ) neuroimaging studies: Statistical power analyses. J Neurosci Methods. 2002; 118: 115-128.

45. Droutman V, Bechara A, Read SJ. Roles of the different sub-regions of the insular cortex in various phases of the decision-making process. Front Behav Neurosci. 2015; 9: 309.

46. Frank GK, Shott ME, DeGuzman MC. Recent advances in understanding anorexia nervosa. F1000Res. 2019; 8. doi: 10.12688/f1000research.17789.1.

47. Brooks SJ, O'Daly OG, Uher R, Friederich HC, Giampietro V, Brammer M, et al. Differential neural responses to food images in women with bulimia versus anorexia nervosa. PLoS One. 2011; 6: e22259.

48. LaBar KS, Gitelman DR, Parrish TB, Kim YH, Nobre AC, Mesulam MM. Hunger selectively modulates corticolimbic activation to food stimuli in humans. Behav Neurosci. 2001; 115: 493500. 
49. Killgore WD, Young AD, Femia LA, Bogorodzki P, Rogowska J, Yurgelun-Todd DA. Cortical and limbic activation during viewing of high- versus low-calorie foods. Neurolmage. 2003; 19: 13811394.

50. Simmons WK, Martin A, Barsalou LW. Pictures of appetizing foods activate gustatory cortices for taste and reward. Cereb Cortex. 2005; 15: 1602-1608.

51. Ely A, Berner LA, Wierenga CE, Kaye WH. Neurobiology of eating disorders: Clinical implications. Psychiatr Times. 2016; 33.

52. Treasure J, Willmott D, Ambwani S, Cardi V, Clark Bryan D, Rowlands K, et al. Cognitive interpersonal model for anorexia nervosa revisited: The perpetuating factors that contribute to the development of the severe and enduring illness. J Clin Med. 2020; 9: 630.

53. Melloni EM, Poletti S, Vai B, Bollettini I, Colombo C, Benedetti F. Effects of illness duration on cognitive performances in bipolar depression are mediated by white matter microstructure. J Affect Disord. 2019; 249: 175-182.

54. Hay $P$, Katsikitis $M$. Emotional responses to images of food in adults with an eating disorder: $A$ comparative study with healthy and clinical controls. Eat Behav. 2014; 15: 371-374.

55. Fairburn CG. Cognitive behaviour therapy and eating disorders. New York: The Guilford Press; 2008.

56. Attia E, Steinglass JE, Walsh BT, Wang Y, Wu P, Schreyer C, et al. Olanzapine versus placebo in adult outpatients with anorexia nervosa: A randomized clinical trial. Am J Psychiatry. 2019; 176: 449-456.

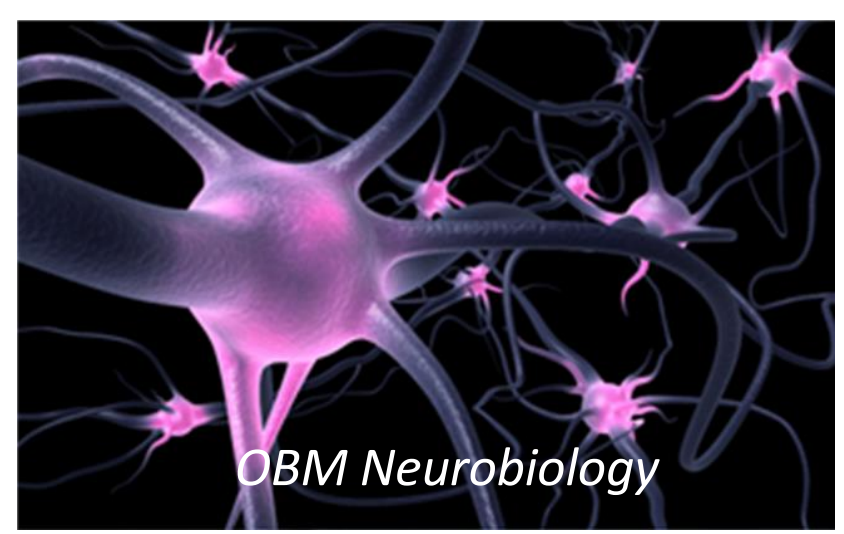

Enjoy OBM Neurobiology by:

1. Submitting a manuscript

2. Joining volunteer reviewer bank

3. Joining Editorial Board

4. Guest editing a special issue

For more details, please visit:

http://www.lidsen.com/journals/neurobiology 